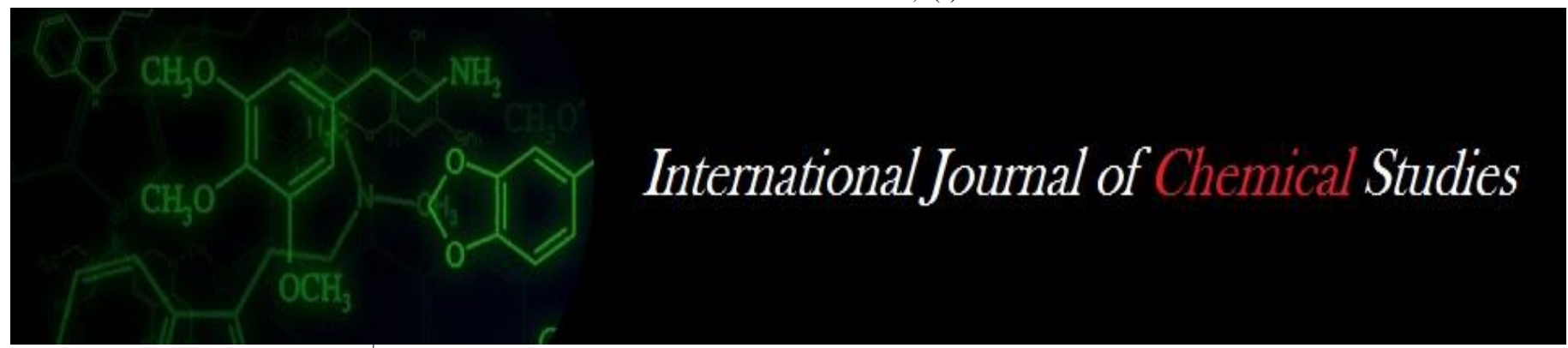

P-ISSN: 2349-8528

E-ISSN: 2321-4902

www.chemijournal.com

IJCS 2021; 9(1): 2643-2646

(C) 2021 IJCS

Received: 07-11-2020

Accepted: 17-12-2020

\section{Shraddha Bansod}

Department of Genetics and

Plant Breeding, Naini

Agricultural Institute, SHUATS,

Prayagraj, Uttar Pradesh, India

Suresh BG

Department of Genetics and

Plant Breeding, Naini

Agricultural Institute, SHUATS

Prayagraj, Uttar Pradesh. India

B Jalandhar Ram

Department of Genetics and

Plant Breeding, Naini

Agricultural Institute, SHUATS,

Prayagraj, Uttar Pradesh, India

GR Lavanya

Department of Genetics and

Plant Breeding, Naini

Agricultural Institute, SHUATS,

Prayagraj, Uttar Pradesh, India

Corresponding Author:

Shraddha Bansod

Department of Genetics and

Plant Breeding, Naini

Agricultural Institute, SHUATS,

Prayagraj, Uttar Pradesh, India

\section{Genetic variability and correlation analysis for yield and yield attributing traits in mungbean [Vigna radiata $(\mathrm{L})$. Wilczek]}

\author{
Shraddha Bansod, Suresh BG, B Jalandhar Ram and GR Lavanya
}

DOI: $\underline{\text { https://doi.org/10.22271/chemi.2021.v9.i1ak.11626 }}$

\begin{abstract}
Twenty mungbean (Vigna radiata (L.) Wilczek) genotypes were evaluated for the estimation of genetic variability parameters, correlation coefficient, path analysis, heritability and genetic advance. The genotypes differed significantly for all the characters. Higher GCV and PCV was observed for the seed index. High heritability coupled with genetic advance as percent of mean showed by harvest index indicating the impact of additive gene expression. The characters days to number of seeds per pod and harvest index showed positive and significant correlation along with positive direct effect on grain yield. Therefore, selection based on this component traits would results improvement in grain yield of mungbean.
\end{abstract}

Keywords: Mungbean (Vigna radiata (L.) Wilczek), genetic variability, heritability, genetic advance, correlation analysis, path coefficient analysis

\section{Introduction}

Mungbean [Vigna radiata (L.) Wilczek] also known as greengram is one of the most important pulse crops of Asia. It is a short duration legume crop belongs to the order Leguminosae and Papilionoideae family. It is a self-pollinating diploid legume with the chromosome number $2 n=2 x=22$ (Karpechenko, 1925). Mung (Greengram) was sown over an area of 4.26 Mha in (kharif + rabi) and recorded a production of $2.01 \mathrm{Mt}$ at and yield level of $472 \mathrm{~kg} / \mathrm{ha}$. During 2017-2018 the total coverage under mungbean. In Uttar Pradesh 0.72 L ha with a production $0.40 \mathrm{Lt}$. and the productivity $555.56(\mathrm{~kg} / \mathrm{ha})$. It can be grown in various crop rotation practices (Singh et al., 2015) because of its short duration nature, wider adaptability, low water requirement and photo insensitiveness. Mungbean is short day, warm season crop, grown mainly in arid and semi-arid regions. It is drought tolerant and has ability to grow under harsh climate and medium to low rainfall situation. It is grown on a variety of soils including black, red lateritic, gravelly and sandy soils. Well drained fertile sandy loam soil with a pH between 6.2- 7.2 is best for mungbean cultivation. Water logged and saline soils are not suitable for mungbean cultivation (Sharma, 2016).

Correlation coefficient analysis is a statistical technique which measures the degree and association between two or more variables. Estimates of correlation coefficient are useful in identifying the component traits which can be used for yield improvement of mungbean. Path coefficient analysis provides a thorough understanding of contribution of various characters by partitioning the correlation coefficient into components of direct and indirect effects (Wright, 1921), which helps the breeder in determining the yield components. To accumulate optimum contribution of yield contributing characters, it is essential to know the association of various characters along with path coefficients (Bhutia et al. 2016) ${ }^{[2]}$. Therefore, present study was conducted to assess correlation and path analysis to identify component traits for developing high yielding varieties of mungbean.

\section{Material and Methods}

The present investigation was carried out at the Field Experimentation Centre, Department of Genetics and Plant Breeding, SHUATS, Prayagraj (U.P.) during Zaid, 2019. The University is situated on the left side of Prayagraj - Rewa National Highway, about $5 \mathrm{~km}$ away from 
Prayagraj city. All types of facilities necessary for cultivation of successful crop including field preparation, inputs and irrigation facilities were provided from the Department of Genetics and Plant Breeding, SHUATS, Prayagraj (U.P.). The experiment was conducted in randomized block design with 20 genotypes the genotypes were replicated 3 times. Genotypes were randomly arranged in each replication divided into 120 plots. The gross area of experiment was 263 $\mathrm{m}^{2}$ and cash plot size was $1 \times 1 \mathrm{~m}$. The row to row spacing was $30 \mathrm{~cm}$ and plant to plant distance was $10 \mathrm{~cm}$. The 5 competitive plants from each of the replication were tagged and observations were taken from these tagged plants at various stages of the crop plant growth. Data were recorded from 12 characters $v i z$, days to $50 \%$ flowering, days to maturity, plant height $(\mathrm{cm})$, number of primary branches per plant, number of cluster per plant, number of pods per plant, pod length $(\mathrm{cm})$, number of seeds per pod, 100 seed weight $(\mathrm{g})$, biological yield(g), harvest index and grain yield per plant(g). mean values were computed data were analysed for analysis of variance as suggested by (Fisher, 1936) and coefficient of variances as well as heritability (in broad sense), as suggested by Burton and Devane (1953). The estimates of genetic advance were obtained by the formula suggested by Lush (1949) and Johnson et al. (1955). Phenotypic and genotypic correlation and path coefficients of variation were computed as per the method given by Dewey and Lu (1959).

\section{Results and Discussion}

The genotypic and phenotypic coefficient of variation, heritability and genetic advance as percent of mean for each of the characters are presented in Table-1. Considerable range in variation was observed for all characters. Phenotypic coefficients of variation values were relatively moderate than corresponding genotypic coefficient of variation for all traits under study. Phenotypic Coefficient of variation (PCV) ranged from number of primary branches per plant $(3.19 \%)$ to seed index (17.33\%). High PCV magnitude was recorded for seed index (17.33) and number of pods per plant (16.16). Moderate values of plant height were also observed by Kumar et al. (2010) ${ }^{[14]}$. Genotypic coefficient of variation which gives the extent of genetic variability in the population, ranged from 2.12 to 14.89 per cent. Moderate genotypic coefficient of variation was observed for seed index (14.89). High phenotypic coefficient of variation and genotypic coefficient of variation were observed for number of primary branches per plant and number of pods per plant. Similar findings were reported by Patel et al. (2014) and Degefa et al. (2014). Indicating the influence of environmental factors less difference was observed between phenotypic and genotypic coefficient of variation in certain cases such as numbers of primary branches per plant, days to $50 \%$ flowering, 100 seed weight, number of seeds per pod, biological yield and days to maturity. Which indicated that these characters were less influenced by the environmental. Similar results also obtained by Kumar et al. (2013) ${ }^{[13]}$. The estimates of heritability (\%) in the broad sense for 12 characters studied, which range from 44 per cent to 98 per cent. High heritability (broad sense) was recorded for characters like harvest index (98) and number of clusters per plant (90). The higher heritability value was registered by the characters under study viz, plant height and number of primary branches per plant. Similar finding was obtained Srivastava and Singh (2012) ${ }^{[22]}$. Genetic advance as $\%$ of mean varied from 2.90 to 29.81 . High genetic advance as \% mean was recorded for harvest index (29.81). The estimated were high genetic advance for number of pods per plant and seed yield per plant was reported by Reddy et al. (2011) ${ }^{[5]}$ and Nizama et al. (2013). Correlation coefficient analysis among grain yield and its contributing characters are shown in Table 2 . There was positive, significant and strong correlation of this trait with number of pods per cluster, number of pods per plant, number of seed index and harvest index at both genotypic and phenotypic levels. Similar results were reported by Aqsa et al. (2010), Tabasum et al. (2010), and Reddy et al. (2011) ${ }^{[19]}$ for seed yield with number of pods per plant, number of pods per cluster, number of clusters per plant, number of seeds per pod. Ahmad et al. (2013) also observed positive genotypic association of seed yield with number of clusters per plant, number of pods per plant. Kumar et al. (2013) ${ }^{[13]}$ and Narasimhulu et al. (2013) ${ }^{[17]}$ also found with number of seeds per pod. Khan et al. (2001) ${ }^{[12]}$, Ahmad et al. (2013) Bhutia et al. (2016) ${ }^{[2]}$ and Choudhary et al. (2016) similar results found for harvest index at phenotypic level. Path coefficient analysis was carried out by taking grain yield as dependent variable to partition the correlation coefficient into direct and indirect effects in order to determine the contribution of different characters towards the grain yield. Direct and positive effect on grain yield per plant was observed for characters like days to $50 \%$ flowering, number of primary branches per plant, number of pods per plant, pod length, plant height, number of seeds per pod, 100 seed weight, biological yield and harvest index. Similar results were reported by Kapadia et al. (2015) ${ }^{[10]}$, Bhutia et al. (2016) ${ }^{[2]}$ and. Days to maturity and number of primary branches per plant had negative direct effect on seed yield per plant. Similar findings were reported by Choudhary et al. (2016) in mungbean. Hence, presently study reveals that number of pods per plant, biological yield, harvest index, number of seeds per pod, 100 seed weight and pod length are important agronomic traits as they have directly contributed towards grain yield, plant height and days to 50 per cent flowering also had direct effect on seed yield. Therefore, more emphasis should be given to these components during selection for higher yield. The interrelationship among yield components would help in increasing the yield levels.

Table 1: Genetic parameters for 12 characters in mungbean

\begin{tabular}{|c|c|c|c|c|c|c|c|c|}
\hline Sr. No & Character & VG & VP & GCV & PCV & $\mathbf{h}^{2} \mathbf{b s}$ & GA & GA as \% mean \\
\hline 1 & Days to 50\% flowering & 3.39 & 6.47 & 4.92 & 6.79 & 52 & 2.75 & 7.34 \\
\hline 2 & Days to maturity & 1.87 & 4.25 & 2.12 & 3.19 & 44 & 1.87 & 2.90 \\
\hline 3 & Plant height & 22.89 & 33.71 & 8.44 & 10.24 & 67 & 8.124 & 14.32 \\
\hline 4 & Primary branches/ plant & 0.08 & 0.21 & 9.59 & 15.11 & 40 & 0.38 & 12.55 \\
\hline 5 & No. of clusters/ plant & 0.48 & 0.53 & 13.15 & 13.84 & 90 & 1.36 & 25.74 \\
\hline 6 & No. of pods/ plant & 2.49 & 5.45 & 10.92 & 16.16 & 45 & 2.19 & 15.21 \\
\hline 7 & Pod length & 0.13 & 0.27 & 4.84 & 7.09 & 46 & 0.50 & 6.81 \\
\hline 8 & No. of seeds/ pod & 0.50 & 0.67 & 6.63 & 7.62 & 75 & 1.28 & 11.89 \\
\hline 9 & 100 seed weight & 0.24 & 0.32 & 14.89 & 17.33 & 73 & 0.86 & 26.34 \\
\hline 10 & Biological yield & 3.54 & 4.42 & 10.45 & 11.67 & 80 & 3.47 & 19.27 \\
\hline
\end{tabular}




\begin{tabular}{|c|c|c|c|c|c|c|c|c|}
\hline 11 & Harvest index & 17.57 & 17.92 & 14.61 & 14.76 & 98 & 8.55 & 29.81 \\
\hline 12 & Grain yield/ plant & 0.13 & 0.18 & 7.18 & 8.34 & 74 & 0.65 & 12.76 \\
\hline \multicolumn{9}{|c|}{$\mathrm{VP}=$ Phenotypic variance } \\
\hline \multicolumn{2}{|c|}{$\begin{array}{l}\text { GCV=Genotypic coefficient of variation } \\
h^{2}(B S)=\text { Heritability broad sense }\end{array}$} & \multicolumn{7}{|c|}{$\begin{array}{l}\mathrm{PCV}=\text { Phenotypic coefficient of variation } \\
\mathrm{GA}=\text { Genetic advance }\end{array}$} \\
\hline
\end{tabular}

Table 2: Correlation coefficient between yield and its related traits in mungbean genotypes at genotypic level

\begin{tabular}{|c|c|c|c|c|c|c|c|c|c|c|c|c|c|}
\hline $\begin{array}{l}\text { Sr. } \\
\text { No }\end{array}$ & Character & $\begin{array}{c}\text { Days to } \\
50 \% \\
\text { flowering }\end{array}$ & $\begin{array}{c}\text { Days to } \\
\text { maturity }\end{array}$ & $\begin{array}{c}\text { Plant } \\
\text { height }\end{array}$ & $\begin{array}{c}\text { Primary } \\
\text { branches } \\
\text { / Plant }\end{array}$ & \begin{tabular}{|l|} 
No. of. \\
clusters \\
/ plant
\end{tabular} & $\begin{array}{l}\text { No. of. } \\
\text { pods / } \\
\text { Plant }\end{array}$ & $\begin{array}{l}\text { Pod } \\
\text { length }\end{array}$ & $\begin{array}{c}\text { No. of. } \\
\text { seeds/ } \\
\text { pod }\end{array}$ & $\left|\begin{array}{c}100 \text { Seed } \\
\text { weight }\end{array}\right|$ & $\begin{array}{c}\text { Biological } \\
\text { yield }\end{array}$ & $\begin{array}{c}\text { Harvest } \\
\text { index }\end{array}$ & $\begin{array}{c}\text { Grain } \\
\text { yield / } \\
\text { plant }\end{array}$ \\
\hline 1 & $\begin{array}{l}\text { Days to } 50 \% \\
\text { flowering }\end{array}$ & 1 & $0.297 *$ & $-0.681 * *$ & -0.003 & -0.089 & $0.263^{*}$ & -0.188 & 0.155 & -0.134 & -0.118 & -0.176 & $-0.551 * *$ \\
\hline 2 & Days to maturity & & 1 & -0.217 & $0.435 * *$ & 0.129 & 0.097 & -0.026 & $0.645^{* *}$ & $-0.598 * *$ & $-0.487 * *$ & $0.479 * *$ & 0.189 \\
\hline 3 & Plant height & & & 1 & $0.283^{*}$ & -0.193 & -0.229 & $0.264 *$ & -0.004 & 0.06 & 0.104 & -0.119 & -0.019 \\
\hline 4 & $\begin{array}{c}\begin{array}{c}\text { Primary branches/ } \\
\text { plant }\end{array} \\
\end{array}$ & & & & 1 & $0.380 * *$ & $0.461 * *$ & $-0.282 *$ & $0.550 * *$ & $-0.749 * *$ & 0.214 & -0.222 & -0.129 \\
\hline 5 & No. of. Clusters / plant & & & & & 1 & $0.917 * *$ & $-0.453 * *$ & 0.121 & -0.181 & 0.089 & -0.011 & 0.12 \\
\hline 6 & No. of. Pods / plant & & & & & & 1 & $-0.651 * *$ & -0.107 & $-0.387 * *$ & 0.228 & -0.138 & 0.06 \\
\hline 7 & Pod length & & & & & & & 1 & 0.007 & $0.295^{*}$ & $-0.655 * *$ & $0.569 * *$ & 0.152 \\
\hline 8 & No. of. Seeds /pod & & & & & & & & 1 & $-0.512 * *$ & -0.013 & 0.177 & $0.347 * *$ \\
\hline 9 & 100 seed weight & & & & & & & & & 1 & $-0.395 * *$ & 0.159 & $-0.304 *$ \\
\hline 10 & Biological yield & & & & & & & & & & 1 & $-0.890 * *$ & -0.071 \\
\hline 11 & Harvest index & & & & & & & & & & & 1 & $0.621 * *$ \\
\hline
\end{tabular}

Table 3: Correlation coefficient between yield and its related traits in Mungbean genotypes at phenotypic level

\begin{tabular}{|c|c|c|c|c|c|c|c|c|c|c|c|c|c|}
\hline $\begin{array}{l}\text { Sr. } \\
\text { No }\end{array}$ & Characters & $\begin{array}{c}\text { Days to } \\
50 \% \\
\text { flowering }\end{array}$ & $\begin{array}{r}\text { Days to } \\
\text { maturity }\end{array}$ & $\begin{array}{c}\text { Plant } \\
\text { height }\end{array}$ & \begin{tabular}{|l} 
Primary \\
branche \\
s/ plant
\end{tabular} & $\begin{array}{l}\text { No. of } \\
\text { cluster } \\
\text { s/plant }\end{array}$ & \begin{tabular}{|c|} 
No. of \\
pods/pl \\
ant
\end{tabular} & $\begin{array}{c}\text { Pod } \\
\text { length }\end{array}$ & $\begin{array}{c}\text { No. of } \\
\text { seeds/po } \\
\text { d }\end{array}$ & $\begin{array}{c}100 \text { seed } \\
\text { weight }\end{array}$ & $\begin{array}{l}\text { Biologic } \\
\text { al yield }\end{array}$ & $\begin{array}{c}\text { Harvest } \\
\text { index }\end{array}$ & \begin{tabular}{|l|} 
Grain \\
yield/ \\
plant \\
\end{tabular} \\
\hline 1 & Days to $50 \%$ flowering & 1 & 0.203 & $-0.27 *$ & -0.051 & -0.114 & 0.178 & 0.005 & 0.106 & -0.019 & -0.081 & -0.118 & $-0.347 * *$ \\
\hline 2 & Days to maturity & & 1 & -0.025 & 0.162 & 0.098 & -0.060 & -0.055 & $0.341 * *$ & $-0.336 * *$ & $-0.355 * *$ & $0.311 *$ & 0.093 \\
\hline 3 & Plant height & & & 1 & 0.152 & -0.152 & -0.001 & $0.257^{*}$ & -0.037 & 0.181 & 0.062 & -0.096 & -0.021 \\
\hline 4 & Primary branches/ plant & & & & 1 & 0.252 & \begin{tabular}{|l|}
0.244 \\
\end{tabular} & -0.141 & $0.281 *$ & $-0.315^{*}$ & 0.107 & -0.168 & -0.009 \\
\hline 5 & No. of clusters/ plant & & & & & 1 & $0.59 * * *$ & $-0.295 *$ & 0.098 & -0.151 & 0.071 & -0.009 & 0.121 \\
\hline 6 & No. of Pods/plant & & & & & & 1 & -0.107 & -0.014 & -0.037 & 0.113 & -0.123 & -0.019 \\
\hline 7 & Pod length & & & & & & & 1 & 0.048 & $0.349 * *$ & $-0.345^{* *}$ & $0.368 * *$ & -0.014 \\
\hline 8 & No. of Seeds/ pod & & & & & & & & 1 & $-0.367 * *$ & -0.083 & 0.129 & $0.255^{*}$ \\
\hline 9 & 100 seed weight & & & & & & & & & 1 & $-0.268 *$ & 0.116 & -0.237 \\
\hline 10 & Biological yield & & & & & & & & & & 1 & $-0.77 * * *$ & -0.111 \\
\hline 11 & Harvest index & & & & & & & & & & & 1 & $0.546 * *$ \\
\hline
\end{tabular}

Table 4: Direct and indirect effects between yield and its different traits in Mungbean genotypes at genotypic level

\begin{tabular}{|c|c|c|c|c|c|c|c|c|c|c|c|c|c|}
\hline $\begin{array}{l}\text { Sr. } \\
\text { No }\end{array}$ & Characters & \begin{tabular}{|c|} 
Days to \\
$50 \%$ \\
Flowering \\
\end{tabular} & $\begin{array}{l}\text { Days to } \\
\text { Maturity }\end{array}$ & $\begin{array}{c}\text { Plant } \\
\text { Height } \\
(\mathrm{cm})\end{array}$ & $\begin{array}{l}\text { Primary } \\
\text { Branches } \\
\text { per Plant }\end{array}$ & $\begin{array}{c}\text { Clusters } \\
\text { per } \\
\text { plant }\end{array}$ & \begin{tabular}{|c|} 
Pods \\
per \\
Plant \\
\end{tabular} & \begin{tabular}{|c|} 
Pod \\
Length \\
in
\end{tabular} & $\begin{array}{c}\text { Seeds } \\
\text { per pod }\end{array}$ & $\begin{array}{c}100 \text { Seed } \\
\text { Weight }\end{array}$ & $\begin{array}{l}\text { Biologic } \\
\text { al yield }\end{array}$ & $\begin{array}{c}\text { Harvest } \\
\text { Index }\end{array}$ & \begin{tabular}{|c} 
Grain \\
Yield per \\
plant
\end{tabular} \\
\hline 1 & $\begin{array}{c}\text { Days to } 50 \% \\
\text { Flowering }\end{array}$ & -0.0185 & -0.0055 & 0.0126 & 0.0001 & 0.0017 & -0.0049 & 0.0035 & -0.0029 & 0.0025 & 0.0022 & 0.0032 & $-0.551 * *$ \\
\hline 2 & Days to maturity & -0.1087 & -0.3661 & 0.0795 & -0.1591 & -0.0476 & -0.0353 & 0.0097 & -0.2361 & 0.219 & 0.1781 & -0.1752 & 0.189 \\
\hline 3 & Plant Height & -0.1782 & -0.0568 & 0.2615 & 0.0741 & -0.0505 & -0.06 & 0.069 & -0.001 & 0.0157 & 0.0271 & -0.031 & -0.019 \\
\hline 4 & $\begin{array}{l}\text { Primary Branches per } \\
\text { Plant }\end{array}$ & 0.0022 & -0.2666 & -0.1737 & -0.6133 & -0.2329 & -0.2827 & 0.1729 & -0.3372 & 0.4595 & -0.1315 & 0.1359 & -0.129 \\
\hline 5 & Clusters per Plant & 0.0024 & -0.0035 & 0.0053 & -0.0104 & -0.0273 & -0.025 & 0.0124 & -0.0033 & 0.0049 & -0.0024 & 0.0003 & 0.12 \\
\hline 6 & Pods per Plant & 0.062 & 0.0228 & -0.0541 & 0.1087 & 0.2163 & 0.2358 & -0.1535 & -0.0252 & -0.0913 & 0.054 & -0.0326 & 0.06 \\
\hline 7 & Pod Length & 0.0154 & 0.0022 & -0.0216 & 0.023 & 0.037 & 0.0532 & -0.0817 & -0.0006 & -0.0241 & 0.0535 & -0.0465 & 0.152 \\
\hline 8 & Seeds per pod & 0.0583 & 0.2429 & -0.0015 & 0.207 & 0.0456 & -0.0403 & 0.0025 & 0.3766 & -0.1929 & -0.0049 & 0.0667 & $0.347 * *$ \\
\hline 9 & 100 Seed Weight & 0.0704 & 0.3153 & -0.0316 & 0.395 & 0.0955 & 0.204 & -0.1557 & 0.2701 & -0.5271 & 0.2082 & -0.0838 & $-0.304^{*}$ \\
\hline 10 & Biological Yield & -0.1373 & -0.5652 & 0.1204 & 0.249 & 0.1032 & 0.2659 & -0.7605 & \begin{tabular}{|l|}
-0.0152 \\
\end{tabular} & -0.4588 & 1.1617 & -1.0336 & -0.071 \\
\hline 11 & Harvest Index & -0.3194 & 0.87 & -0.2157 & -0.4026 & -0.0207 & -0.2513 & 1.0338 & 0.3217 & 0.2889 & -1.617 & 1.8174 & $0.621 * *$ \\
\hline
\end{tabular}

Table 5: Direct and indirect effects between yield and its different traits in Mungbean genotypes at phenotypic level

\begin{tabular}{|c|c|c|c|c|c|c|c|c|c|c|c|c|c|}
\hline & Character & \begin{tabular}{|c|} 
Days to \\
$50 \%$ \\
Flowering
\end{tabular} & $\begin{array}{c}\text { Days to } \\
\text { Maturity }\end{array}$ & $\begin{array}{c}\text { Plant } \\
\text { height }\end{array}$ & \begin{tabular}{|l|} 
Primary \\
Branches \\
per Plant
\end{tabular} & $\begin{array}{c}\text { Clusters } \\
\text { per } \\
\text { plant }\end{array}$ & \begin{tabular}{|c|} 
Pods \\
per \\
Plant
\end{tabular} & $\begin{array}{c}\text { Pod } \\
\text { Length }\end{array}$ & $\begin{array}{c}\text { Seeds } \\
\text { per pod }\end{array}$ & $\begin{array}{c}100 \\
\text { Seed } \\
\text { Weight }\end{array}$ & $\begin{array}{c}\text { Biological } \\
\text { yield }\end{array}$ & $\begin{array}{c}\text { Harvest } \\
\text { Index }\end{array}$ & $\begin{array}{l}\text { Grain } \\
\text { Yield per } \\
\text { plant }\end{array}$ \\
\hline 1 & \begin{tabular}{|l|} 
Days to \\
\end{tabular} & & & & & 0.019 & -0.0296 & & & & & & $-0.347 * *$ \\
\hline 2 & & & & & & & & & & & & & \\
\hline 3 & & & & & & & & & & & & & \\
\hline 4 & $\begin{array}{l}\text { Primary Branches per } \\
\text { Plant }\end{array}$ & 0.0011 & -0.0035 & -0.0032 & -0.0213 & -0.0054 & $\mid-0.0052$ & 0.003 & -0.006 & 0.0067 & -0.0023 & 0.0036 & -0.009 \\
\hline
\end{tabular}




\begin{tabular}{|c|c|c|c|c|c|c|c|c|c|c|c|c|c|}
\hline 5 & Clusters per Plant & 0.0063 & -0.0054 & 0.0084 & -0.0139 & -0.055 & -0.0325 & 0.0163 & -0.0054 & 0.0083 & -0.0039 & 0.0005 & 0.121 \\
\hline 6 & Pods per Plant & 0.0153 & -0.0052 & -0.0002 & 0.021 & 0.0507 & 0.0858 & -0.0092 & -0.0012 & -0.0032 & 0.0097 & -0.0106 & -0.019 \\
\hline 7 & Pod Length & -0.0013 & 0.0122 & -0.0561 & 0.0309 & 0.0645 & 0.0233 & -0.2179 & -0.0106 & -0.0762 & 0.0754 & -0.0802 & -0.014 \\
\hline 8 & Seeds per pod & 0.0209 & 0.0675 & -0.0073 & 0.0556 & 0.0195 & -0.0028 & 0.0096 & 0.1975 & -0.0725 & -0.0165 & 0.0255 & $0.255^{*}$ \\
\hline 9 & 100 Seed Weight & 0.0024 & 0.0417 & -0.0225 & 0.0391 & 0.0188 & 0.0046 & -0.0433 & 0.0455 & -0.124 & 0.0333 & -0.0144 & -0.2378 \\
\hline 10 & Biological Yield & -0.0491 & -0.2141 & 0.0378 & 0.0647 & 0.0428 & 0.0683 & -0.2082 & -0.0504 & -0.1615 & 0.6019 & -0.4686 & -0.111 \\
\hline 11 & Harvest Index & -0.1317 & 0.3462 & -0.107 & -0.1878 & -0.0107 & -0.137 & 0.4095 & 0.1438 & 0.1292 & -0.8663 & 1.1127 & $0.546^{* *}$ \\
\hline
\end{tabular}

\section{Acknowledgement}

We are thankful to the Honorable Vice Chancellor, HOD, Teaching and non-Teaching staff of the Department of Genetics and Plant Breeding, Naini Agricultural Institute, Sam Higginbottom University of Agriculture, Technology and Sciences, Prayagraj- 211007, U.P., for providing all necessary facilities and support.

\section{References}

1. Anand G, Anandhi K, Paulpandi VK. Genetic variability, correlation and path analysis for yield and yield components in $\mathrm{F} 6$ families of greengram (Vigna radiata (L) Wilczek) under rainfed condition. Electronic Journal of Plant Breeding, Article 2016 DOI: 10.5958/0975928X 2016.00054.5.

2. Bhutia P, Lal GM, Thomas N. Studies on genetic variability, correlation and path analysis in green gram [Vigna radiata (L.) Wilczek] germplasm. International Journal of Agriculture Science 2016;8(51):2267-2272.

3. Bisht N, Singh DP, Khulbe RK. Genetic variability and correlation studies in advance inter- specific and inter varietal lines and cultivars of mungbean [Vigna radiate (L.) Wilczek] Journal of Food Legume 2014;27(2):155157.

4. Das A, Biswas M, Ghosh KK. Genetic divergence in greengram (Vigna radiate L. Wilczek). Agronomy Journal. ISSN 2014, 1812-5379.

5. Reddy DKR, Venkateswarlu O, Obaiah MC, Jyothi GLS. Studies on genetic variability, character association and path-coefficient analysis in greengram (Vigna radiata (1) Wilczek). Legume Research 2011;34(3):202-206.

6. Eswaran R, Senthil Kumar N. Correlation and path analysis in greengram [Vigna radiate (L.) Wilczek] for drought stress, Plant Archives 2015;15(1):247-249.

7. Gadakh SS, Dethe AM, Kathale MN. Genetic variability, correlations and path analysis studies on yield and its components in mungbean [Vigna radiata (L.) Wilczek]. Bioinfolet 2013;10(2a):441-447.

8. Hemavathy AT, Shunmugavalli N, Anand G. Genetic variability, correlation and path co-efficient studies on yield and its components in mungbean [Vigna radiata (L.) Wilczek) Legume Research 2015;38(4):442-446.

9. Jagdhane NM, Suresh BG, Ram BJ, Yadav P. Genetic variability and character association for seed yield in Mungbean [Vigna radiata (L.) Wilczek]. Journal of Pharmacognosy and Phytochemistry 2017;6(4):13881390.

10. Katiyar M, Kumar S, Kumar N. Path analysis, association and variation of grain yield attributes in mung bean [Vigna radiata $(\mathrm{L}$.$) Wilczek]. International Journal of$ Advanced Research A 2015;3(6):2410-2413.

11. Kapoor R, Lavanya GR, Suresh Babu G. Estimation of genetic variability in mungbean (Vigna radiata (L) Wilczek). Research on crops 2005;6(3):509-510.

12. Khanpara MD, Vachhani JH, Jivani LLS, Vaghasia PM. Correlation and path coefficient analysis in greengram
[Vigna radiata (L.) Wilczek]. Asian Journal of Biological Science 2012;(7):34-38.

13. Kumar A, Lavanya GR, Reddy AP, Babu GS. Genetic variation for seed yield and characters association in Greengram. J Agric. Res. Technol 2013;38(2):321-323.

14. Kumar S, Kerkhi SA, Sirohi A. Pooran Chand Studies on genetic variability, heritability and character association in induced mutants of mungbean (Vigna radiata (L.) Wilczek) Progressive Agriculture 2010;10(2):365-367.

15. Lal M, Singh D. Utilization of genetic diversity and its association characters in mungbean (Vigna radiata $\mathrm{L}$. Wilczek). Legume Research 2014;37(6):679-681.

16. Makeen K, Garard A, Arif J, Singh AK. Genetic variability and correlations studies on yield and its components in mungbean (Vigna radiata (L.) Wilczek). J Agron 2007;6(1):216-218.

17. Narasimhulu R, Naidu NV, Shanthi Priya M, Rajarajeswari V, Reddy KHP. Indian J Plant Sciences 2013;2(3):2319-3824.

18. Nazir A, Sadiq MS, Hanif M, Abbas G, Haidar S. Genetic parameters and path analysis in mungbean, [Vigna radiata (L.) Wilczek]. J Agricultural Research, Lahore 2005;43(4):339-347.

19. Reddy KD, Venkateswarlu O, Obaiah MC, Siva Jyothi GL. Studies on genetic variability, character association and path co-efficient analysis in Greengram [Vigna radiata. (L.) Wilczek]. Legume Research 2011;34(3):202-206.

20. Reddy VLN, Reddisekhar M, Reddy KR, Reddy KH. Genetic variability for yield and its components in mungbean, [Vigna radiata (L.) Wilczek]. Legume Research 2003;26(4):300-302, 1852.

21. Singh M, Singh TP, Sharma SK, Thakur HL. Influence of cropping system on combining ability and gene action for grain yield and its components in green gram (Vigna radiata). Indian Journal. Agriculture. Science 2010;73:356-357.

22. Srivastava RL, Singh G. Genetic variability, correlation and path analysis in mungbean [Vignaradiata (L.) Wilczek]. Indian Journal of Life Science 2012;2(1):6165.

23. Tejbir Singh, Amitesh S, Alie FA. Morpho-physiological traits as selection criteria for yield improvement in Mungbean [Vigna radiata (L.) Wilczek]. Legume Research 2009;32(1):36-40.

24. Verma Hari Kesh et al. Genetic Variability, Correlation and Path Analysis in Mungbean [Vigna radiata (L.) Wilczek] International Journal of Curren Microbiology and Applied Sciences International Journal of Current Microbiology and Applied Sciences Volume 2017, 21662173.

25. Zaid IU, Hussain IK, Sajid K. Genetic variability and correlation analysis for yield components in mungbean Vigna radiata L. Wilczek ARPN $\mathrm{J}$ of Agric. and Biological Science 2012;7(11):885-891. 